\title{
A scopic review on the diagnostic dilemmas and newer treatment modalities in Hemophagocytic Lymphohistiocytosis
}

\author{
Vinod Paul ${ }^{1}$, vyas rathaur ${ }^{2}$, Amanta Ittoop $^{3}$, Rajkumar Sananganba ${ }^{1}$, Nowneet Bhat ${ }^{1}$, and \\ Monika Pathania ${ }^{1}$ \\ ${ }^{1}$ All India Institute of Medical Sciences - Rishikesh \\ ${ }^{2}$ AIIMS Rishikesh \\ ${ }^{3}$ All India Institute of Medical Sciences, Rishikesh
}

October 1, 2020

\begin{abstract}
Abstract: Hemophagocytic lymphohistiocytosis is a rare and fatal systemic illness arising secondary to an immune dysregulation .Primary HLH is due to genetic defects and secondary HLH is caused due to unchecked macrophage recruitment following an acquired trigger. It is often diagnosed late in view of its rarity and similarities of presentation to sepsis and SIRS. A compelete curative solution to this problem is hematopoietic stem cell transplant, though secondary cases are often seen to have sustained remission with immune-chemotherapy COVID 19 has also been postulated to cause a HLH like scenario with blunted NK cell number and function. Recent advances in this field comprising of various immunosuppressant based regimens, myeloablative therapies preceding stem cell transplant and improved techniques of stem cell transplant have improved the outcomes. Here we try to present the pathogenesis, etiology, diagnostic criteria and the dilemmas, various treatment strategies, prognostic markers and the most recent researches regarding this rare disease.
\end{abstract}

\section{TITLE PAGE}

Type of article: Review article

Title of the article: A scopic review on the diagnostic dilemmas and newer treatment modalities in Hemophagocytic Lymphohistiocytosis

Running title- Recent Diagnostics and management of HLH

Contributors:-

1) Dr Vinod Paul, MD, DNB ,Senior resident, Department of pediatrics, AIIMS Rishikesh, email - vinodpaulchully1@gmail.com

2) Dr Vyas Kumar Rathaur, MD. Associate professor, department of pediatrics, AIIMS Rishikesh

3) Dr Amanta Lucy Ittoop, MBBS, Junior resident, Department of Anaesthesia, , AIIMS

Rishikesh, email- lucyamanta@gmail.com

4) Dr Rajkumar Sananganba, MBBS, Junior Resident, Department of Pediatrics, AIIMS Rishikesh, emailrksana@outlook.com

5) Dr Nowneet Kumar Bhat, MD, Professor and HOD, Department of Pediatrics, AIIMS Rishikesh, emaildrnmbhat@gmail.com 
6) Dr Monika Pathania, MD. Associate professor, department of internal medicine, AIIMS

Rishikesh. , email-monica.rims@gmail.com

Corresponding author

Dr Vyas Kumar Rathaur

E-mail address : vyasrathaur@gmail.com

Address : AIIMS Rishikesh

Phone number- 8126021558

Total no of tables- 3

Word counts

For abstracts- 152

For the article text- 3499

Source of support- self

Presentation at a meeting- nil

Conflict of interest- none

Acknowledgement- we sincerely thank the Department of paediatrics and Hemat-oncology for their continuous support in management of our children. We are thankful to the residents

sincerely involved in the treatment of all our patients.

\begin{tabular}{ll}
\hline Abbreviations & \\
\hline HLH & Hemophagocytic Lymphohistiocytosis \\
SIRS & Systemic inflammatory response syndrome \\
EBV & Epstein Barr virus \\
APC & Antigen presenting cell \\
CTL & Cyto-toxic lymphocyte \\
SLE & Systemic lupus erythematosus \\
JIA & Juvenile idiopathic arthritis \\
CRP & C-reactive protein \\
MODS & Multi-organ dysfunction \\
TNF & Tumour necrosis factor \\
\hline
\end{tabular}

Title: A scopic review on the diagnostic dilemmas and newer treatment modalities in Hemophagocytic Lymphohistiocytosis

Abstract:

Hemophagocytic lymphohistiocytosis is a rare and fatal systemic illness arising secondary to an immune dysregulation .Primary HLH is due to genetic defects and secondary HLH is caused due to unchecked macrophage recruitment following an acquired trigger. It is often diagnosed late in view of its rarity and similarities of presentation to sepsis and SIRS. A compelete curative solution to this problem is hematopoietic stem cell transplant, though secondary cases are often seen to have sustained remission with immune-chemotherapy COVID 19 has also been postulated to cause a HLH like scenario with blunted NK cell number and function. Recent advances in this field comprising of various immunosuppressant based regimens, myeloablative therapies preceding stem cell transplant and improved techniques of stem cell transplant have improved the 
outcomes. Here we try to present the pathogenesis, etiology, diagnostic criteria and the dilemmas, various treatment strategies, prognostic markers and the most recent researches regarding this rare disease.

Introduction :

Hemophagocytic lymphohistiocytosis (HLH) is an immune dysregulation syndrome which is caused due to defect of the Natural killer (NK) cells. There are 2 variants - primary (familial) and secondary. Primary $\mathrm{HLH}$ is caused due to mutations of certain genes which leads to improper activation and functioning of NK cells leading to an uncontrolled cytokine storm. Secondary HLH is secondary to infections (e.g.: EBV, Dengue), rheumatological issues and malignancy.

History:

HLH was first noted way back in 1939, when Scott and Robb Smith construed this condition to be an atypical presentation of Hodgkin's lymphoma. However, post mortem histopathology showed histiocytic infiltration in the bone marrow with phagocytosed erythrocytes. Hence they named it histiocytic medullary reticulosis (HMR). ${ }^{1}$ It was later in 1959 that similar findings were identified in pediatric population by Farquhar and Claireaux. Two siblings presented with the same during infancy and rapidly progressed into multi organ failure leading to death in both. ${ }^{2}$

Pathophysiology:

In a normal body NK cells and cytotoxic T cells (CTLs) identify the Antigen presenting cells (APC dendritic cells and histiocytes) and then release perforin and granzyme in a well-regulated fashion through a newly formed synapse called immunological synapse (IS). ${ }^{3}$ Perforins and granzymes are normally stored in granules in lysosomes of NK cells and CTLs. ${ }^{4}$ Limited amounts of required Inflammatory mediators are also transported across the IS towards the APCs. Perforins form pores on the APCs and later granzymes help in apoptosis. ${ }^{4}$ Together all these processes orchestrate in a well regulated manner to first destroy the antigen and then set stage for apoptosis. ${ }^{3}$ Following apoptosis, the dying cells secrete enzymes which stimulate each other in a cascade fashion to destroy the IS, hence controlling the release of inflammatory mediators. ${ }^{5}$ Any hindrance in this pathway gives a longer life to the IS causing more uncontrolled and unnecessary release of inflammatory mediators - cytokines, tumour necrosis factor (TNF) and interferons. This inflammatory cocktail stimulates macrophages and turns on the secretion of IL-6. ${ }^{5}$ The unchecked Macrophages hence activated, phagocytose the other blood cells causing cytopeneias. ${ }^{6}$ Also the antigens presented by the antigen presenting cells, are not effectively killed causing persistent activation of these cells and leading to further recruitment of NK cells and immunologic synapses, thus leading to further hypercytokinemia (cytokine storm). ${ }^{7}$ The macrophages and histiocytes with the phagocytosed cells, infiltrate various organs, impeding their function like liver, spleen, and lymph nodes leading to hepatosplenomegaly and lymphadenopathy.

Etiology:

Familial HLH:

The incidence of familial (primary) HLH was seen to be 1.2 in 1000000 children per year, according to a study conducted in $2009 .^{8}$ An Indian meta - analysis review showed that the incidence of primary HLH in India is 1 in $50000 .{ }^{9}$ They are inherited in an autosomal recessive pattern. There are 5 main gene loci identified currently, which could cause familial HLH - a genetic loci on chromosome 9, PFR1, UNC13D, STX11, STXBP2. (Table 1)

Most common mutations noted are those of PRF1 and UNC13D causing FHL2 and FHL3 respectively. Together they account for $70 \%$ of all the familial HLH cases. ${ }^{10}$ Age of presentation is mostly during early infancy . ${ }^{11}$

Other genetic diseases - Griscelli syndrome, Chediak Higashi, Hermansky-Pudlak and X linked lymphoproliferative disease (type 1 and 2 ) could also pre-dispose to HLH. ${ }^{12,13,14}$ (Table 2)

Secondary HLH: 
This is caused due to conditions which initiates NK cells or cytotoxic T lymphocytes induced inflammation. NK cells constitute the early defenders against intracellular pathogens such as viruses. Hence viruses are the most common infectious agents leading to HLH. ${ }^{8}$ In a meta-analysis by Srinivas Rajagopala and Navneet singh it was found that viral infections were the most common cause of Secondary HLH. EBV and dengue were the most common viruses to cause HLH in children. In the current period, it is worth mentioning that many of the deaths occurring due to COVID 19 are also due to severe HLH like cytokine storm. ${ }^{9,15}$ COVID 19 causes blunting of NK cell number and functions leading to improper clearance of the APCs and unchecked elevation in tissue damaging inflammatory mediators. ${ }^{10}$ Leishmaniasis, Ricketsia, Malaria, Histoplasmosis, Enteric fever, and Tuberculosis were also responsible agents in adults. The other causes were connective tissue disorders such as stills disease, juvenile idiopathic arthritis (JIA) and SLE. ${ }^{9}$ Malignancies especially those affecting $\mathrm{T}$ cells - like anaplastic $\mathrm{T}$ cell lymphoma and acute lymphoblastic lymphoma were seen to cause HLH in a study by Lehmberg et al in $2015 .{ }^{16}$ Few B cell lineage neoplasias like hodgkins lymphoma were also seen. Post bone marrow transplant, a HLH like scenario could arise.

Diagnosis:

Diagnosis of this condition is based on symptoms, signs, and markers of inflammation. Prolonged high grade fever is present in $90-100 \%$ of the children, and hence HLH is one of the differential diagnosis of Pyrexia of unknown origin. ${ }^{17}$ Splenomegaly is also seen in more than $80 \%$ of children according to all the studies reviewed ${ }^{11,17,18}$ and lymphadenopathy is seen in approximately $50 \%$ of cases. ${ }^{19}$ The child at presentation appears very sick with features mimicking sepsis and septic shock ( tachycardia, tachypnea, hyperpyrexia and occasionally hypotension ). Pancytopenia or bicytopenia is always present because of significant phagocytosis, and the differential count always show lymphocyte predominance. Neutropenia is occasionally noted. CRPH which is a Marker of inflammation is seen to be very high. Activated histiocytes bind with factor 10, hence activating the common pathway of coagulation and consuming fibrinogen leading to hypofibrinogenemia. ${ }^{20}$ ESR is hence low, since erythrocyte sedimentation is dependent on fibrinogen. Ferritin is elevated because ferroportin mediated iron efflux increases due to increase in growth differentiation factor 15 during the hyperinflammation in HLH. Inflammation also causes upregulation of hemoxygenase - which breaks heme to iron. Other markers of inflammation which increase are IL-6, IL-18, IFN-gamma, TNF-alpha. Hypertriglyceridemia occurs due to inhibition of lipo-protien lipase by TNF alpha. ${ }^{21}$

Diagnostic criteria for HLH was formulated in 2004 and confirms the diagnosis if any of the gene abnormalities can be detected ( molecular diagnosis ) or any 5 of the 8 below mentioned criteria are fulfilled:

1. Fever $>38.5$ degree centigrade

2. Splenomegaly

3. Cytopenia (at least 2 cell lines): Platelet $<100000 / \mathrm{microL}$

a) $\mathrm{Hb}<9$;

b) Absolute neutrophil count $<1000$ c)

4) Hypertriglyceridemia $>265 \mathrm{mg} / \mathrm{dl}$

5) Hemophagocytosis in bone marrow, spleen, lymph node

6) Low or absent NK cell activity

7) Ferritin > 500ng/ml

8) Elevated soluble CD25 ( IL-2 receptor - on T cell ) ${ }^{20}$

These diagnostic criteria was formulated according to prevalence of these features in 369 patients in HLH study of 2004 . More than $70 \%$ of children in this study showed all these features. ${ }^{22}$

Ferritin levels for diagnosis of HLH is a very debated criteria. Though $500 \mathrm{ng} / \mathrm{ml}$ is considered significant, various authors have opined about increasing the threshold for confirmation of the diagnosis. Ferritin levels of $10000 \mathrm{ng} / \mathrm{ml}$ was considered significant by Allen et al and $6000 \mathrm{ng} / \mathrm{ml}$ by Belfike et al. ${ }^{23,24}$ CD107a was seen to be upregulated in all activated NK cells and hence was considered to be a marker of NK cell activity. 
As mentioned earlier, infiltration of macrophages and histiocytes in various organs cause multi organ failure.

Liver involvement is seen in most of the children with HLH. Hence HLH is considered to be a differential diagnosis of Acute Liver Failure (ALF) during the first year of life. In a study on 251 children with HLH in Korea, $86 \%$ of the children were seen to have hepatomegaly, with $63 \%$ having transaminitis and $35 \%$ showing icterus. Around $27 \%$ of them had prolonged APTT and hypofibrinogenemia was seen in $62 \%$ of the children. ${ }^{19}$ Histology of affected liver shows lymphohistiocytic infiltration in the portal system, along with bile duct injury. However lobular histology is preserved. Kupffer cell hyperplasia is also noted along with sinusoidal congestion with histiocytes, along with hemosiderosis. However liver biopsy is usually avoided due to the catastrophic bleeds which may ensue. ${ }^{25}$

Symptoms and signs of lung infiltration are very difficult to be differentiated from other infections. ${ }^{12}$

CNS infiltration is seen in mostly the familial variant, and its symptoms are varied ranging from neck rigidity due to meningeal involvement to irritability due to encephalitis. Three stages were described by Henter and Nennesmo in 1997, where stage 1 showed only meningeal infiltration in the mildest form and stage 3 showed diffuse infiltration of parenchyma, meninges, along with multifocal tissue necrosis. ${ }^{26}$ Features of raised ICP - bulged fontanelle, vomiting, blurring of vision are also seen. Convulsions also are occasionally seen when parenchyma is infiltrated. Cranial nerves 6 and 7 are occasionally involved. ${ }^{12}$

It must be noted that the diagnosis of HLH is mostly made with progression of symptoms, not responding to the usual therapies, and hence the commoner possibilities must always be considered first. SIRS, sepsis induced multi-organ dysfunction (MODS), and macrophage activation syndrome (MAS) secondary to JIA share the same clinical and laboratory features as that of HLH. This dilemma leads to over diagnosis of HLH. ${ }^{27}$ Arico et al hence made an algorithm with three tests - perforin expression ( by flow cytometry ), lymphocyte 2B4 receptor function (antibody dependent cellular cytotoxicity assay ) and NK cell activity. ${ }^{28}$ In centers which do not have such facilities- supportive therapies for sepsis and MODS should be initiated and chemotherapy for HLH should be reserved for children who do not respond to them. ${ }^{27}$ However delay in treatment of HLH is also a determinant of mortality.

Treatment:

The most important goal in treatment is to arrest the stormy hyperinflamatory response within the body and to promote apoptosis of the antigen presenting cell to prevent further inflammatory cells' recruitment. Etoposide is a chemotherapeutic drug which does both the above functions. Once this is achieved- the familial, persistent or relapse disease is treated with hemopoeitic stem cell transplant (HSCT). ${ }^{22}$

To achieve appropriate immune suppression HLH 94 consensus had come up with dxamethasone and etoposide regimen for 8 weeks and cyclosporine was added from the 9th week. Intrathecal methotrexate was given to children with CNS involvement. However HLH 2004 study suggested addition of cyclosporine in the begining itself along with dexamethasone and etoposide. CNS involvement was treated with intrathecal methotrexate and hydrocortisone as per 2004 guidelines. There were marginal improvement in the survival with HLH 2004 as compared to HLH 94 guidelines - $62 \%$ with the former and $54 \%$ with the latter. ${ }^{22}$ In $\mathrm{v} / \mathrm{o}$ of significant complications of cyclosporine (renal toxicity and posterior reversible encephalopathy syndrome) many experts do not start early cyclosporine therapy, though some clinicians opine that it helps in maintaining remission for longer time. ${ }^{29}$ Early initiation of steroids after ruling out malignancy must be considered even before all investigation reports are obtained. ${ }^{29}$ Treatment should be tailored according to the disease severity and liver and renal functions. Children who show a very rapid response should be weaned off faster. Dexamethasone which is started at $10 \mathrm{mg} / \mathrm{m}^{2}$ could also be tapered down as per severity either weekly or once in two weeks. ${ }^{29}$ At the same time any flare in the disease warrants further intensification of both steroid dose and etoposide frequency.

Anti thymocyte globulin (ATG), which causes T cell lymphopenia, has also been used along with steroids instead of etoposide and has shown to have good efficacy with lesser degree of cytopenia. A study done by Mahlaoui et al from showed this regimen to have complete remission in $73 \%$ and partial remission in $24 \%$ of 
the 38 children enrolled..$^{30}$

Intravenous immunoglobulins could be used in the treatment of HLH as a primary agent or an adjunct. In a multi centre study conducted by Larroche $\mathrm{C}$ et al it was noticed that 17 children $(9$ primary and 8 secondary) showed remission to 2 IV-IG doses of $1.6 \mathrm{~g} / \mathrm{kg}$ per dose. They also mention about the importance of early delivery of the same, and report inadequate results with late administration. ${ }^{31}$ Also few Indian studies Divya nandhakumar et al and Sarala Rajajee et al showed good response to IVIG with steroid regimens in secondary HLH - 17 children in the former and 19 children in the latter studies achieved remission. These studies have used IV IG in secondary HLH only. ${ }^{32,33}$ However few studies such as Imashuku et al suggest that IV IG based primary regimens are not adequate in treating HLH. ${ }^{34}$

EBV induced HLH has shown good response to addition of Rituximab (anti CD 20 antibody) and Anakinra (IL1 recptor antagonist) was found to be beneficial as an early add on in Systemic onset juvenile idiopathic arthritis induced HLH. ${ }^{35,36} \mathrm{HLH}$ like presentation following bone marrow transplant can be treated by adalimumab - a TNF alpha inhibitor. ${ }^{37}$ (Table 3)

Response to therapy could be monitored using ferritin levels or soluble IL2 receptor concentration. A drop of $15 \%$ of the former within first 48 hours and any decrease in the latter is considered to be a positive response. ${ }^{29}$ Decrease in triglyceride level also suggest improvement. ${ }^{21}$

Refractory cases (nil response after 8 weeks standard therapy) could be treated with Alemtuzumab (anti CD 52 antibody) along with steroids, etoposide and cyclosporine as shown in Marsh et al. $1 \mathrm{mg} / \mathrm{kg}$ of alemtuzumab was given as an add on to 14 children and $25 \%$ of them showed partial remission. ${ }^{38}$ Doxorubicin (liposomal), Etoposide and Methylprednisolone (DEP) is another regimen used as a salvage therapy especially in adult refractory cases. ${ }^{39}$

Hematopoietic stem cell transplant (HSCT):

HSCT for this disease was first carried out in 1995, and advances from then in this field have led to better outcomes. The stem cells are usually harvested from peripheral blood $(78 \%)$ or bone marrow (22\%) of the donor. ${ }^{40}$ Umblical cord stem cells have also been used for transplant and have shown good results with better survival in infants with primary HLH. ${ }^{41}$ However this cannot be done in bigger children and adults, since umblical cord may not have sufficient stem cells. Finding a fully matched donor for HSCT could be difficult, and hence partially matched family member's stem cells are used in few centers - haploidentical HSCT. The chances of the donor also having the abnormal gene causing HLH is high in such cases and if so the recipient would go into relapse. ${ }^{38}$

Following HSCT, it takes 10-20 days for engraftment to occur. If all hematopoietic cells post-transplant are of donor origin, then the recipient is called a complete chimera and shows complete chimerism. 20-30\% stable chimerism is required for a successful transplant, and to keep the child in sustained remission. ${ }^{38} \mathrm{~A}$ donor chimerism of $20 \%$ means that following the transplant, $20 \%$ of the cells in the marrow and the circulating blood of the recipient would be that of the donor origin. Increasing level of recipient cells could lead to graft rejection, and the opposite may result in the complication of graft vs host disease (GVHD). During mixed chimerism, whether an increased recipient origin cells indicates graft failure or a graft, recipient peaceful coexistence is difficult to assert. ${ }^{42}$ Hence clinical monitoring of remission, and early identification of GVHD is prime following the procedure.

Myelo-ablative conditioning regimens are nowadays being used to kill the recipient marrow cells, so that there is enough room for the donor cells in the recipient marrow. The advent of this therapy, also known as conditioning, preceding transplant has improved the outcomes in HLH quite significantly. Initially Busulphan and cyclophosphamide were used. However adverse effects such as veno-occlusive disorders ensued leading to adverse outcomes. ${ }^{38}$ Better outcomes were noticed with reduced intensity conditioning regimens like melphalan and fludarabine, or treosulfan and thiotepa, though at the expense of higher rates of mixed chimerism. The results were further enhanced with addition of serotherapy with alemtuzumab which is a monoclonal antibody of CD52 ( present on all mature lymphocytes, but absent in the stem cells ). ${ }^{43}$ 
Alemtuzumab could be given at the proximal, distal or intermediate phase of the regimen. Data from various studies favor both proximal and intermediate phase administration. Mixed chimerism is seen in such cases but with adequate tolerance, because the levels of alemtuzumab is seen to be high enough till around 60 days. ${ }^{44}$ Also Alemtuzumab mediated depletion of T cells and APCs bring down the inflammation prior to HSCT - improving the chances of survival. However it is to be noted that low donor chimerism lesser than $20 \%$ warrants post HSCT cell therapy, which could be either donor lymphocyte infusion (DLI) or a second HSCT.

GVHD is a dreaded complication of HSCT. In acute GVHD, occurring within 100 days, fever, rash, hepatitis with hyperbilirubinemia, vomiting, diarrhoea, abdominal pain and weight loss are the presenting complaints. Chronic GVHD affects the skin predominantly with lichenoid rash and sclerotic changes. Hepatobiliary system is also affected and significant immunodeficiency occur frequently. Cyclosporine and methylprednisolone are tailored into the HSCT regimen, starting 2-3 days prior to transplant. Cyclosporine is usually continued for upto 100 days post-transplant. Methotrexate and tacrolimus could also be used.

Post-transplant, another cytokine storm can occur which looks like the original HLH itself. This condition is seen to have good response to adalimumbab. ${ }^{37}$

Carl et al from United states of America, studied 36 children with HLH underwent stem cell transplant, following reduced intensity conditioning with melphalan and fludarabine, with intermediate alemtuzumab. The 1 year overall survival was $82.4 \%$ and 18 months overall survival of $68 \%$ was noted. ${ }^{45}$ Another study by Katharina et al on 60 children who underwent HSCT for HLH showed similar promising results with 5 year overall survival at $75 \%$, though $30 \%$ of them required secondary post HSCT cell therapy (DLI or second HSCT). Both melphalan and treosulfan based regimens were used for conditioning with serotherapy given in the proximal limb of therapy with alemtuzumab and ATG. All the regimens were found to be equally efficacious. ${ }^{46}$

\section{Outcomes :}

Children with this condition do not survive more than 2 months without treatment. ${ }^{11}$ Younger age, CNS involvement, severe elevation in transaminase, severe cholestasis, and coagulation abnormalities are considered to be indicators of poor outcomes according to a study by Koh et al. ${ }^{18}$ Outcomes are also negative if the treatment is delayed, and hence at least steroids should be commenced immediately after ruling out all other possibilities such as malignancy. In some children repeated, multiple blood and plasma exchange have shown positive response, and have been used to buy some time till diagnosis is confirmed. ${ }^{47}$ Hematopoietic stem cell transplant is the final curative treatment available, and in familial cases, this must be performed as soon as possible during remission. Hence HLA typing and searching an appropriate donor is commenced as soon as the disease is confirmed in such cases. Having active disease during transplant, using haploidentical stem cells and a poor match in HLA typing were seen to have high rates of failure following HSCT. ${ }^{38,46}$

Recent updates and research:

Pre B cell colony enhancing factor (PBEF) - a marker of severe inflammation, has been shown to be a very good marker for diagnosis and disease activity. This has shown a better specificity because with remission it touches the baseline levels, as compared to IL2 receptor levels which stays mildly elevated for some time. ${ }^{48}$

Hybrid immunotherapy is a new treatment modality which is being tried in few centers where steroid, etoposide and ATG are being used. Other regimens with the primary agent being alemtuzumab and tocilizumab (IL-6 receptor inhibitor) are being studied in France and Philadelphia respectively. However there are no published results from these studies.

Cytokines cause the inflammatory storm through Janus kinase (JAK1,2) and signal transducer and activator of transcription (STAT) associated receptors. Ruxolitinib causes inhibition of JAK1,2 receptors with amelioration of inflammation in murine models. ${ }^{49}$ This agent as primary agent is being tried in Michigan. Anti IFN-gamma antibodies - Emapalumab is also being tried. A study on 13 children treated with Emapalumab showed improvement of parameters in 9 children and 7 of them went for HSCT. ${ }^{50}$ 
Hematopoietic stem cell gene therapy with selected cells of high level perforin expression led to better survival in animal model studies. Perforin gene transfer using lenti virus as vector into progenitor cells of perforin deficient mice was performed and significant reduction in cytokine secretion in mice, with more than $30 \%$ engraftment, was noted..$^{51}$

In another study Micro RNA -126 (enhancer of perforin expression) was placed in the perforin DNA loci of the stem cells, and was transplanted into perforin deficient mice. These mice showed a dose dependent increase in survival and better clearance of pathogen. ${ }^{52}$ Such gene therapy if successful in humans could improve the outcome remarkably.

Extracorporeal adsorption technique has shown success in adults. It was used in 2 adults with HLH secondary to herpes infection, and was found to be effective in bringing the cytokines' level down. An adsorption cartridge made up of polystyrene divinylbenzene copolymer beads is used, which binds to hydrophobic compounds with a molecular weight of $10-55 \mathrm{kDa} .{ }^{53}$

Conclusion:

HLH is a fatal storm of inflammation in the body, which mimics other severe illnesses such as SIRS and sepsis. Differentiating between them clinically is a challenge. Treatment of both sepsis and HLH are also very different with former being treated with broad spectrum antibiotics and latter with immunosuppressants. The recent diagnostic guidelines have led to an over diagnosis of HLH leading to flare up of infections and missing malignancies at an earlier stage due to the immunosuppressants used for the same. At the same time delaying the treatment of HLH also causes mortality. This dilemma warrants immediate but prudent treatment with exercise of cautious monitoring.

References:

1) Scott RB, Robb-Smith AH. Histiocytic medullary reticulosis. The Lancet. 1939 Jul 22;234(6047):194-8.

2) Farquhar JW, Claireaux AE. Familial haemophagocytic reticulosis. Archives of Disease in Childhood. 1952 Dec;27(136):519.

3) Jenkins MR, Rudd-Schmidt JA, Lopez JA, Ramsbottom KM, Mannering SI, Andrews DM, Voskoboinik I, Trapani JA. Failed CTL/NK cell killing and cytokine hypersecretion are directly linked through prolonged synapse time. Journal of Experimental Medicine. 2015 Mar 9;212(3):307-17.

4) Janka GE, Lehmberg K. Hemophagocytic lymphohistiocytosis: pathogenesis and treatment. Hematology. 2013 Dec 6;2013(1):605-11.

5) Esteban YM, de Jong JL, Tesher MS. An overview of hemophagocytic lymphohistiocytosis. Pediatric annals. 2017 Aug 15;46(8):e309-13.

6) Grzybowski B, Vishwanath VA. Hemophagocytic lymphohistiocytosis: A diagnostic conundrum. Journal of pediatric neurosciences. 2017 Jan;12(1):55.

7) Usmani GN, Woda BA, Newburger PE. Advances in understanding the pathogenesis of HLH. British journal of haematology. 2013 Jun;161(5):609-22.

8) Filipovich AH. Hemophagocytic lymphohistiocytosis (HLH) and related disorders. Hematology. 2009 Jan 1;2009(1):127-31.

9) Rajagopala S, Singh N. Diagnosing and treating hemophagocytic lymphohistiocytosis in the tropics: systematic review from the Indian subcontinent. Acta medica academica. 2012 Oct 4;41(2):161-74.

10) Sieni E, Cetica V, Mastrodicasa E, Pende D, Moretta L, Griffiths G, Aricò M. Familial hemophagocytic lymphohistiocytosis: a model for understanding the human machinery of cellular cytotoxicity. Cellular and Molecular Life Sciences. 2012 Jan 1;69(1):29-40. 
11) Henter JI, Aricò M, Elinder G, Imashuku S, Janka G. Familial hemophagocytic lymphohistiocytosis: primary hemophagocytic lymphohistiocytosis. Hematology/oncology clinics of North America. 1998 Apr 1;12(2):417-33.

12) Malinowska I, Machaczka M, Popko K, Siwicka A, Salamonowicz M, Nasiłowska-Adamska B. Hemophagocytic syndrome in children and adults. Archivum immunologiae et therapiae experimentalis. 2014 Oct $1 ; 62(5): 385-94$.

13) Zhou S, Ma H, Gao B, Fang G, Zeng Y, Zhang Q, Qi G. Characterization of a novel disease-causing mutation in exon 1 of SH2D1A gene through amplicon sequencing: a case report on HLH. BMC Medical Genetics. 2017 Dec;18(1):1-7.

14) Basiaga ML, Weiss PF, Behrens EM. BIRC4 mutation: an important rare cause of uveitis. Journal of clinical rheumatology: practical reports on rheumatic \& musculoskeletal diseases. 2015 Dec;21(8):444.

15) van Eeden C, Khan L, Osman MS, Cohen Tervaert JW. Natural Killer Cell Dysfunction and Its Role in COVID-19. International Journal of Molecular Sciences. 2020 Jan;21(17):6351.

16) Lehmberg K, Sprekels B, Nichols KE, Woessmann W, Müller I, Suttorp M, Bernig T, Beutel K, Bode SF, Kentouche K, Kolb R. Malignancy-associated haemophagocytic lymphohistiocytosis in children and adolescents. British journal of haematology. 2015 Aug;170(4):539-49.

17) Astigarraga I, Gonzalez-Granado LI, Allende LM, Alsina L. Haemophagocytic syndromes: The importance of early diagnosis and treatment. Anales de Pediatria (English Edition). 2018 Aug 1;89(2):124-e1.

18) Koh KN, Im HJ, Chung NG, Cho B, Kang HJ, Shin HY, Lyu CJ, Yoo KH, Koo HH, Kim HJ, Baek HJ. Clinical features, genetics, and outcome of pediatric patients with hemophagocytic lymphohistiocytosis in $\mathrm{K}$ orea: report of a nationwide survey from $\mathrm{K}$ orea $\mathrm{H}$ istiocytosis $\mathrm{W}$ orking $\mathrm{P}$ arty. European journal of haematology. 2015 Jan;94(1):51-9.

19) Verma SP, Naik R, Basu D, Vinod KV, Kar R, Dutta TK. Hemophagocytic Lymphohistiocytosis in Adults and Adolescents-Experience from a Tertiary Care Centre in South India. Nat J Lab Med. 2017;6:1001-5.

20) Ooe K. Pathogenesis of hypofibrinogenemia in familial hemophagocytic lymphohistiocytosis. Pediatric pathology. 1991 Jan 1;11(4):657-61.

21) Okamoto M, Yamaguchi H, Isobe Y, Yokose N, Mizuki T, Tajika K, Gomi S, Hamaguchi H, Inokuchi K, Oshimi K, Dan K. Analysis of triglyceride value in the diagnosis and treatment response of secondary hemophagocytic syndrome. Internal Medicine. 2009;48(10):775-81.

22) Bergsten E, Horne A, Arico M, Astigarraga I, Egeler RM, Filipovich AH, Ishii E, Janka G, Ladisch S, Lehmberg K, McClain KL. Confirmed efficacy of etoposide and dexamethasone in HLH treatment: long-term results of the cooperative HLH-2004 study. Blood. 2017 Dec 21;130(25):2728-38.

23) Allen CE, Yu X, Kozinetz CA, McClain KL. Highly elevated ferritin levels and the diagnosis of hemophagocytic lymphohistiocytosis. Pediatric blood \& cancer. 2008 Jun;50(6):1227-35.

24) Belfeki N, Strazzulla A, Picque M, Diamantis S. Extreme hyperferritinemia: etiological spectrum and impact on prognosis. Reumatismo. 2019;71(4):199-202.

25) Padhi S, Sarangi R, Patra S, Samal SC. Hepatic Involvement in Hemophagocytic Lymphohistiocytosis. InHepatobiliary Diseases 2019 Nov 28. IntechOpen.

26) Henter JI, Nennesmo I. Neuropathologic findings and neurologic symptoms in twenty-three children with hemophagocytic lymphohistiocytosis. The Journal of pediatrics. 1997 Mar 1;130(3):358-65.

27) Castillo L, Carcillo J. Secondary hemophagocytic lymphohistiocytosis and severe sepsis/systemic inflammatory response syndrome/multiorgan dysfunction syndrome/macrophage activation syndrome share 
common intermediate phenotypes on a spectrum of inflammation. Pediatric Critical Care Medicine. 2009 May 1;10(3):387-92.

28) Arico M, Allen M, Brusa S, Clementi R, Pende D, Maccario R, Moretta L, Danesino C. Haemophagocytic lymphohistiocytosis: proposal of a diagnostic algorithm based on perforin expression. British journal of haematology. 2002 Oct;119(1):180-8.

29) Marsh RA, Haddad E. How i treat primary haemophagocytic lymphohistiocytosis. British journal of haematology. $2018 \mathrm{Jul} ; 182(2): 185-99$.

30) Mahlaoui N, Ouachee-Chardin M, de Saint Basile G, Neven B, Picard C, Blanche S, Fischer A. Immunotherapy of familial hemophagocytic lymphohistiocytosis with antithymocyte globulins: a single-center retrospective report of 38 patients. Pediatrics. 2007 Sep 1;120(3):e622-8.

31) Larroche C, Bruneel F, Andre MH, Bader-Meunier B, Baruchel A, Tribout B, Genereau T, Zunic P. Intravenously administered gamma-globulins in reactive hemaphagocytic syndrome. Multicenter study to assess their importance, by the immunoglobulins group of experts of CEDIT of the AP-HP. InAnnales de medecine interne 2000 Nov (Vol. 151, No. 7, pp. 533-539).

32) Nandhakumar D, Loganatha A, Sivasankaran M, Sivabalan S, Munirathnam D. Hemophagocytic lymphohistiocytosis in children. The Indian Journal of Pediatrics. 2020 Feb 14:1-6.

33) Rajajee S, Ashok I, Manwani N, Rajkumar J, Gowrishankar K, Subbiah E. Profile of hemophagocytic lymphohistiocytosis; efficacy of intravenous immunoglobulin therapy. The Indian Journal of Pediatrics. 2014 Dec 1;81(12):1337-41.

34) Imashuku S, Tabata Y, Teramura T, Hibi S. Treatment strategies for Epstein-Barr virus-associated hemophagocytic lymphohistiocytosis (EBV-HLH). Leukemia \& lymphoma. 2000 Jan 1;39(1-2):37-49.

35) Chellapandian D, Das R, Zelley K, Wiener SJ, Zhao H, Teachey DT, Nichols KE, EBV-HLH Rituximab Study Group, Hale G, Minkov M, Karlhuber S. Treatment of E pstein B arr virus-induced haemophagocytic lymphohistiocytosis with rituximab-containing chemo-immunotherapeutic regimens. British journal of haematology. 2013 Aug;162(3):376-82.

36) Eloseily EM, Weiser P, Crayne CB, Haines H, Mannion ML, Stoll ML, Beukelman T, Atkinson TP, Cron RQ. Benefit of anakinra in treating pediatric secondary hemophagocytic lymphohistiocytosis. Arthritis \& Rheumatology. 2020 Feb;72(2):326-34.

37) Noguchi M, Moritake H, Kamimura S, Sonoda M, Ishimura M, Inagaki J. Adalimumab for treatment of hemophagocytic syndrome following unrelated bone marrow transplantation in a boy with Behcet's disease and secondary myelodysplastic syndrome. Bone marrow transplantation. 2018 Sep;53(9):1214-7.

38) Lehmberg K, Moshous D, Booth C. Haematopoietic Stem Cell Transplantation for Primary Haemophagocytic Lymphohistiocytosis. Frontiers in pediatrics. 2019;7:435.

39) Wang Y, Huang W, Hu L, Cen X, Li L, Wang J, Shen J, Wei N, Wang Z. Multicenter study of combination DEP regimen as a salvage therapy for adult refractory hemophagocytic lymphohistiocytosis. Blood, The Journal of the American Society of Hematology. 2015 Nov 5;126(19):2186-92.

40) Machowicz R, Suarez F, Jedrzejczak WW, Eikema DJ, de Wreede LC, Blok HJ, Isaksson C, Einsele H, Poire X, Nikolousis M, Johansson JE. Allogeneic hematopoietic stem cell transplantation in hemophagocytic lymphohistiocytosis (HLH) in adults: a retrospective study of the chronic malignancies and inborn errors working parties (CMWP and IEWP) of the EBMT.

41) Ohga S, Kudo K, Ishii E, Honjo S, Morimoto A, Osugi Y, Sawada A, Inoue M, Tabuchi K, Suzuki N, Ishida Y. Hematopoietic stem cell transplantation for familial hemophagocytic lymphohistiocytosis and Epstein-Barr virus-associated hemophagocytic lymphohistiocytosis in Japan. Pediatric blood \& cancer. 2010 Feb;54(2):299-306 
42) Liesveld JL, Rothberg PG. Mixed chimerism in SCT: conflict or peaceful coexistence?. Bone marrow transplantation. 2008 Sep;42(5):297-310.

43) Marsh RA, Rao MB, Gefen A, Bellman D, Mehta PA, Khandelwal P, Chandra S, Jodele S, Myers KC, Grimley M, Dandoy C. Experience with alemtuzumab, fludarabine, and melphalan reduced-intensity conditioning hematopoietic cell transplantation in patients with nonmalignant diseases reveals good outcomes and that the risk of mixed chimerism depends on underlying disease, stem cell source, and alemtuzumab regimen. Biology of Blood and Marrow Transplantation. 2015 Aug 1;21(8):1460-70.

44) Morris EC, Rebello P, Thomson KJ, Peggs KS, Kyriakou C, Goldstone AH, Mackinnon S, Hale G. Pharmacokinetics of alemtuzumab used for in vivo and in vitro T-cell depletion in allogeneic transplantations: relevance for early adoptive immunotherapy and infectious complications. Blood. 2003 Jul 1;102(1):404-6.

45) Allen CE, Marsh R, Dawson P, Bollard CM, Shenoy S, Roehrs P, Hanna R, Burroughs L, Kean L, Talano JA, Schultz KR. Reduced-intensity conditioning for hematopoietic cell transplant for HLH and primary immune deficiencies. Blood. 2018 Sep 27;132(13):1438-51.

46) Wustrau K, Greil J, Sykora KW, Albert MH, Burkhardt B, Lang P, Meisel R, Wossmann W, Beier R, Schulz A, Bader P. Risk factors for mixed chimerism in children with hemophagocytic lymphohistiocytosis after reduced toxicity conditioning. Pediatric Blood \& Cancer. 2020 Sep;67(9):e28523

47) Ladisch S, Ho W, Matheson D, Pilkington R, Hartman G. Immunologic and clinical effects of repeated blood exchange in familial erythrophagocytic lymphohistiocytosis

48) Gao ZY, Li XY, Bhandari V, Li LD, Lan D. Pre-B-cell colony-enhancing factor is markedly elevated in childhood hemophagocytic lymphohistiocytosis. Genet Mol Res. 2015 May 18;14(2):18.

49) Maschalidi S, Sepulveda FE, Garrigue A, Fischer A, de Saint Basile G. Therapeutic effect of JAK1/2 blockade on the manifestations of hemophagocytic lymphohistiocytosis in mice. Blood, The Journal of the American Society of Hematology. 2016 Jul 7;128(1):60-71.

50) Daver N, McClain K, Allen CE, Parikh SA, Otrock Z, Rojas-Hernandez C, Blechacz B, Wang S, Minkov M, Jordan MB, La Rosee P. A consensus review on malignancy-associated hemophagocytic lymphohistiocytosis in adults. Cancer. 2017 Sep 1;123(17):3229-40.

51) Carmo M, Risma KA, Arumugam P, Tiwari S, Hontz AE, Montiel-Equihua CA, Alonso-Ferrero ME, Blundell MP, Schambach A, Baum C, Malik P. Perforin gene transfer into hematopoietic stem cells improves immune dysregulation in murine models of perforin deficiency. Molecular Therapy. 2015 Apr 1;23(4):737-45.

52) Tiwari S, Hontz A, Terrell CE, Arumugam P, Carmo M, Risma K, Jordan M, Malik P. High level of perforin expression is required for effective correction of hemophagocytic lymphohistiocytosis. Human gene therapy. 2016 Oct 1;27(10):847-59.

53) Frimmel S, Hinz M, Schipper J, Bogdanow S, Mitzner S, Koball S. Cytokine adsorption is a promising tool in the therapy of hemophagocytic lymphohistiocytosis. The International journal of artificial organs. 2019 Nov;42(11):658-64.

Table 1: Genes involved in primary $\mathbf{H L H}$

\begin{tabular}{lll}
\hline Gene & Protein & Function \\
\hline Genetic loci- $9^{\text {th }}$ chromosome & Unknown & Unknown \\
PFR1 & Perforin & Cytolysis and immune regulation \\
UNC13D & MUNC13-4 & Prime cytolytic granules \\
STX11 & Syntaxin & Transport cytolytic granules \\
STXBP & Syntaxin binding protien & Fuse cytolytic granules to cell membrane, and release perforin \\
\hline
\end{tabular}


Table 2: Genetic abnormalities associated with HLH

\begin{tabular}{|c|c|c|c|}
\hline Condition & Gene affected & Function affected & Associated feature \\
\hline Griscelli & RAB27A & $\begin{array}{l}\text { Uncontrolled } \mathrm{T} \text { cell and } \\
\text { macrophage activation; } \\
\text { Defective docking }\end{array}$ & Albinism \\
\hline Chediak Higashi & LYST & $\begin{array}{l}\text { Defective biogenesis of } \\
\text { cytolytic granules }\end{array}$ & Albinism \\
\hline Hermansky Pudlak & AP3B1 & $\begin{array}{l}\text { Defective transport of } \\
\text { cytolytic granules }\end{array}$ & Albinism \\
\hline $\begin{array}{l}\text { X-linked } \\
\text { lymphoproliferative } \\
\text { disorder - } 1\end{array}$ & SH2D1A, & $\begin{array}{l}\text { Signaling lymphocytic } \\
\text { activation } \\
\text { molecule-associated } \\
\text { protein (SAP) deficiency- } \\
\text { inappropriate } \\
\text { lymphocyte recruitment }\end{array}$ & $\begin{array}{l}\text { Immunodeficiency } \\
\text { Requires HSCT }\end{array}$ \\
\hline $\begin{array}{l}\mathrm{X} \text { - linked } \\
\text { lymphoproliferative } \\
\text { disorder - } 2\end{array}$ & BIRC4 & Inhibition of apoptosis & $\begin{array}{l}\text { Immunodeficiency Uveitis } \\
\text { (rare) Requires HSCT }\end{array}$ \\
\hline
\end{tabular}

Table 3: Biologicals and its Mechanism of action

\begin{tabular}{lll}
\hline Biologicals used in HLH & Mechanism of action & Specially used in: \\
\hline Rituximab & CD 20 antibody & EBV associated HLH \\
Anakinra & IL-1 receptor antagonist & sJIA associated HLH \\
Alemtuzumab & CD 52 antibody & Serotherapy in HSCT for HLH \\
Tocilizumab & IL-6 receptor antagonist & Trials ongoing \\
Ruxolitinib & JAK 1,2, receptor antagonist & Trials ongoing \\
Emapalumab & IFN gamma antibody & Trials ongoing \\
Adalimumab & TNF alpha inhibitor & Post-transplant associated HLH \\
\hline
\end{tabular}

Legends:

Table 1: Genes involved in primary HLH

Table 2: Genetic abnormalities associated with HLH

Table 3: Biologicals and its Mechanism of action

Keywords

- Hemophagocytic Lymphohistiocytosis

- Immunochemotherapy

- Hematopoietic stem cell transplant

- NK cell

- Myelo-ablative therapy

- Reduced intensity conditioning

\section{Hosted file}

Tables for HLH article.pdf available at https://authorea.com/users/363065/articles/ 483980-a-scopic-review-on-the-diagnostic-dilemmas-and-newer-treatment-modalities-in- 
hemophagocytic-lymphohistiocytosis 